\title{
New Trends and Techniques in Gastroenterology
}

The 12th Annual Meeting of the Japanese Gastroenterological Association (JGA) was organized by Prof. Hideyuki Hiraishi and was held in Tokyo on February 26 and 27, 2016. During this conference, 7 special lectures, 6 educational lectures, 4 core symposia, 13 workshops, and one forum were held among general presentations.

Articles published in the present Digestion JGA Special Issue 2017 represent the best presentations from the 12th Annual Meeting. The session chairpersons and editorial board members recommended numerous papers from their sections; subsequently, the editorial committee of the JGA selected distinguished presentations from these recommendations and invited the authors to submit their papers for this special issue. Four reviews and 7 original articles were accepted for final publication. All manuscripts were independently reviewed by 2 members of the JGA editorial committee.

Advances in endoscopic technology towards the diagnosis and treatment of gastrointestinal cancers have been remarkable in the recent years. In this special issue, one review describes the endoscopic full-thickness resection of gastrointestinal tumors and another describes the evaluation of lymphovascular invasion in rectal neuroendocrine tumors. One original article describes the endoscopic resection of non-ampullary superficial duodenal tumors, whereas another describes the clinical efficacy of colon capsule endoscopy in the detection of laterally spreading tumors.

Alterations in the composition of gut microbiota have been shown in a number of gastrointestinal diseases. Manipulation of gut microbiota represents a novel poten- tial therapeutic strategy for the treatment of gastrointestinal diseases. In this regard, one review focuses on gut microbiota in nonsteroidal anti-inflammatory drug-induced small intestinal damage, whereas one original article describes the effects of the probiotic Lactobacillus gasseri on aspirin-induced small intestinal lesions and another described changes in intestinal motility and gut microbiota composition in a rat stress model.

Helicobacter pylori causes gastritis, peptic ulcer, and gastric cancer. However, the role of other bacteria for gastric pathogenesis has not yet been clarified. An original article of the special issue describes Helicobacters other than $H$. pylori in patients with gastric diseases.

The therapeutic strategy of steroid refractory ulcerative colitis has not yet been established. Therefore, an original article describes the short- and long-term outcomes of infliximab treatment for steroid refractory ulcerative colitis and prognostic factors for outcomes.

In addition, this issue contains a review and an original article describing functional gastrointestinal disorders. The review describes the diagnostic and therapeutic strategies of achalasia subtypes and esophagogastric junction outflow obstruction, and the original article describes gender differences in gastric emptying in healthy volunteers and patients with functional dyspepsia.

We believe this issue will provide useful information to clinicians and investigators. We wish to express our sincere gratitude to all the authors, chairpersons, and editorial board members for their contributions to this special issue.

Yasuhisa Shinomura, $\mathrm{MD}, \mathrm{PhD}$ Chairperson of the JGA Editorial Committee 